\title{
LE CANADA SEMI-DRY
}

\author{
JAMES G. LOVEYS AND FRANK O. WAGNER
}

(Communicated by Andreas R. Blass)

\begin{abstract}
We show that a simple group $G$ of finite Morley Rank acting faithfully on as divisible abelian group must be a linear group over some algebraically closed field.
\end{abstract}

\section{INTRODUCTION}

One of the early conjectures in the theory of stable groups is Cherlin's Conjecture, which states that any simple group of finite Morley rank should be an algebraic group over an algebraically closed field. Reineke [Re] proved that a strongly minimal group is abelian; Cherlin [Ch] showed that a connected rank two group is soluble. In rank three, he proved that if the group contains a subgroup of rank 2, then it is $\mathrm{PSL}_{2}(K)$ for some field $K$. These results were consequently generalised by Chantal Berline to superstable groups of $U$ rank $\leq 3$ [Be] and by Ehud Hrushovski to $p$-simple groups of $p$-weight $\leq 3$, where $p$ is any regular type $[\mathrm{H} 1]$.

Alas, little progress on the original conjecture was made. Hrushovski showed in a series of surprising constructions that one of the original ways of attacking it could not work and that the group might not be pure, i.e., have additional structure and still finite rank. Corredor [C] and Borovik/Poizat [P1] proved that any minimal nonsolvable group of finite rank with only nilpotent subgroups must be quite bad indeed: It is the disjoint union (except for $\{1\}$ ) of a conjugate set of self-normalising maximal subgroups and does not contain any involutions! However, recent work by Ivanov and $\mathrm{Ol}^{\prime}$ shanskii [IO] and Corredor seem to indicate that these things might actually exist.

We shall show that in generalised geometry of characteristic zero, they are at least linear. That is, if $G$ is a simple group of automorphisms of a divisible abelian group $A$, then there is a definable finite-dimensional vector space $V$ over some definable algebraically closed field $K$ and $G$ embeds into $\mathrm{GL}(V)$.

Cela a le goût du Canada Dry, cela a la couleur du Canada Dry-c'est le Canada Semi-Dry! [P2]

Received by the editors December 21, 1990 and, in revised form, April 30, 1991.

1991 Mathematics Subject Classification. Primary 03C45.

Research supported by NSERC and FCAR. 


\section{TRUTH OR CONSEQUENCES}

In the following, all groups will be taken to be infinitely definable, unless otherwise indicated. We shall work in a saturated model of our theory, which is supposed to be superstable of finite $U$-rank. If $X$ acts on $Y, C_{X}(Y)$ denotes the elements of $X$ fixing $Y$ and $C_{Y}(X)$ the elements of $Y$ fixed by $X$.

Definition 1. A quasi-endomorphism of the connected abelian group $A$ is a homomorphism $A \rightarrow A / C$, where $C<A$ is finite.

It can be seen as a subgroup of $A \times A$ such that the projection onto the first coordinate is surjective and finite-to-one.

(In the infinite rank case, we would have to replace finite by hereditarily orthogonal to the generic type of $A$.)

Definition 2. If $S$ is a set of quasi-endomorphisms of the abelian group $A$, then $A$ is $S$-minimal if $A$ possesses no proper infinite $S$-invariant subgroup, that is, there is no infinite $A^{\prime}<A$ such that all $s \in S$ induce a quasi-endomorphism $s \uparrow A^{\prime}=s \cap\left(A^{\prime} \times A^{\prime}\right)$ of $A^{\prime}$.

First we shall show

Theorem 3. If $S$ is an infinite type-definable set of quasi-endomorphisms of the abelian $S$-minimal group $A$ of unbounded exponent, then $A$ is divisible torsionfree.

Proof. Note that by minimality, $A$ is connected and $n A=A$ for all $n<\omega$. Hence by finiteness of rank, $A[n]$ has to be finite for all $n$, being the kernel of the surjective map $x \mapsto n x$. Furthermore, $A$ is divisible. Thus if there is any torsion, the torsion subgroup $T$ of $A$ is infinite.

As $A[n]$ is finite for all $n$, there are only countably many finite subgroups of $A$. Hence, for some finite $C<A$, the definable set $S^{\prime} \subseteq S$ of quasiendomorphisms with cokernel $C$ is infinite. Consider a finite subgroup $A[n]$ of $A$. As a quasi-endomorphism in $S^{\prime}$ maps $A[n]$ to $(A / C)[n]$, there are only finitely many homomorphisms from $A[n]$ to $A / C$ and there are two distinct quasi-endomorphism in $S^{\prime}$ that agree on $A[n]$.

By compactness, there are two distinct quasi-endomorphisms $\sigma$ and $\sigma^{\prime}$ in $S^{\prime}$ that agree on the whole of $T$. But now consider $A^{\prime}=\operatorname{ker}\left(\sigma-\sigma^{\prime}\right)$. This is a proper subgroup of $A$ containing $T$. Thus the minimal infinitely definable supergroup of $T$ is contained in $A^{\prime}$, hence proper in $A$, and obviously $S$ invariant (as $T$ is), contradicting the minimality of $A$.

Therefore, in our context, quasi-endomorphisms are endomorphisms.

Theorem 4. Suppose that there is an infinite type-definable set $S$ of automorphisms of the abelian, torsionfree group $A$, such that $A$ is $S$-minimal. Then there is a subgroup $A_{1} \leq A$ and a field $K$ such that $A_{1} \cong K^{+}$definably. Furthermore, $S$ embeds into a matrix ring over $K$.

Proof. Put $G=\langle S\rangle$, the group of automorphisms of $A$ generated by $S$, and let $A_{1} \leq A$ be a minimal subgroup of $A$. For any $g_{1}, \ldots, g_{n} \in G$, if $A_{1} \not$ $\sum_{i=1}^{n} g_{i} A_{1}$, then $A_{1} \cap \sum_{i=1}^{n} g_{i} A_{1}=\{0\}$ by minimality; hence any such sum of translates of $A_{1}$, written with the minimal number of summands, is direct. By the finiteness of rank, there must be a maximal sum, which must be equal to $A$ 
by minimality. Hence $A=\bigoplus_{i=1}^{n} g_{i} A_{1}$ for some $n<\omega$ and $g_{i} \in G$, and we may assume that $g_{1}=$ id. We write $A_{i}$ for $g_{i} A_{1}$ and $\pi_{i}$ for the projection onto the $i$ th coordinate.

Let $h$ be any element in $G$ and $1 \leq i \leq n$. Then $(h, i)$ determines an automorphism of $A_{1}$ via $\sigma_{(h, i)}(x)=g_{i}^{-1}\left(\pi_{i}(h(x))\right)$. Let $0 \neq a \in A_{1}$ be any element and $X=\left\{\sigma_{(h, i)}(a): 1 \leq i \leq n, h \in \bigcup_{i=1}^{n} g_{i} S g_{i}^{-1}\right\}$. We claim that $X \subset A_{1}$ is infinite.

Indeed, if $\sigma_{(h, i)}(a)=\sigma_{\left(h^{\prime} i\right)}(a)$, then $\operatorname{ker}\left(\sigma_{(h, i)}-\sigma_{\left(h^{\prime}, i\right)}\right)$ contains $a$ and hence is infinite, and thus the identity on $A_{1}$. Putting $h_{j}=g_{j} h g_{j}^{-1}$ and $h_{j}^{\prime}=g_{j} h^{\prime} g_{j}^{-1}$, we see that if $\sigma_{\left(h_{j}, i\right)}(a)=\sigma_{\left(h_{j}^{\prime}, i\right)}(a)$ then for the same reason, $\sigma_{(h, i)}$ and $\sigma_{\left(h^{\prime}, i\right)}$ agree on $A_{j}$. And if this is true for all $1 \leq i \leq n$, then $h$ and $h^{\prime}$ agree on $A$ and are equal. Hence, if $X$ were finite, there would be only finitely many different automorphisms of $A$ in $S$, contradiction.

By the minimality of $A_{1}, X$ is indecomposable and hence generates the whole of $A_{1}$, by Zil'ber's indecomposibility theorem: $A_{1}=\sum_{i=1}^{k} X$ for some $k<\omega$. But now $K$, the ring of definable endomorphisms of $A_{1}$, is definable as a set: If $\sigma \in K$ then $\sigma(a)=b$ for some $b \in A_{1}$. There are $x_{1}, \ldots, x_{k} \in X$ such that $\sum_{i=1}^{k} x_{i}=b$, and if $\sigma_{i}$ is the automorphism corresponding to $x_{i}$, then $\operatorname{ker}\left(\sigma-\sum_{i=1}^{k} \sigma_{i}\right)$ is infinite and hence equals $A_{1}$. Thus $\sigma=\sum_{i=1}^{k} \sigma_{i}$ is uniformly definable.

Note that $K-\{0\}$ is in fact a group of automorphisms: Any kernel, being less than $A_{1}$, has to be $\{0\}$, and any image, being infinite, has to be $A_{1}$ by minimality. Hence $K$ is a division ring; superstability implies that $K$ is an algebraically closed field.

We now want to embed $G$ into $M_{n}(K)$. To this end, consider the map

$$
\varphi: g \mapsto\left(\pi_{i} g \uparrow A_{j}\right)_{1 \leq i, j \leq n}
$$

from $G$ to $M:=\left(K_{i j}\right)_{1 \leq i, j \leq n}$, where $K_{i j}$ is the ring of definable homomorphisms from $A_{j}$ to $A_{i}$. Note that $K_{i i}$ is essentially the same as $K$ and the $K_{i j}$ are affinised versions of $K$ : one has merely forgotten which element is the 1.

Obviously, this map is injective. We have to check that this is a group homomorphism. But easily,

$$
\begin{aligned}
{[\varphi(g) \varphi(h)]_{i j} } & =\sum_{k=1}^{n}\left(\pi_{i} g \uparrow A_{k}\right)\left(\pi_{k} h \uparrow A_{j}\right)=\sum_{k=1}^{n} \pi_{i} g \pi_{k}\left(h \uparrow A_{j}\right) \\
& =\pi_{i} g\left(\sum_{k=1}^{n} \pi_{k}\right)\left(h \uparrow A_{j}\right)=\pi_{i}(g h) \uparrow A_{j}=\varphi(g h)_{i j} .
\end{aligned}
$$

Finally, we can fix isomorphisms from $A_{1}$ to $A_{i}$ (the $g_{i}$ will do) and identify $K$ with $K_{i j}$ via $K \ni k \mapsto g_{i} k g_{j}^{-1} \in K_{i j}$. This induces an isomorphism of $M$ and $M_{n}(K)$. Of course, the elements of $G$, being automorphisms, will map into $\mathrm{GL}_{n}(K)$, and we are done.

Note that although $A$ arid $A_{1}$ have been taken infinitely definable, $K$ will be properly definable, being a field in a superstable theory. Hence a posteriori, $A_{1}$ and $A$ will be definable as well. Indeed, they will even be $\omega$-stable, as 
it follows easily from the Hrushovski decomposition of a superstable group (see e.g. [P1, Chapter 6]) that any divisible abelian group of finite $U$-rank is $\omega$-stable: It is finite-dimensional, and its dimensions are carried in definable minimal quotients of subgroups, which are divisible and hence connected. They are $p$-simple for some regular type $p$ of $U$-rank 1 that cannot be trivial; if $p$ does not have Morley rank 1, it is locally modular by a result of Buechler [B], and by [H2], there is a properly infinitely definable subgroup whose generic is domination-equivalent to $p$, contradicting minimality.

But now $M$, being $K$-internal, is also $\omega$-stable, and so is $S$. However, $G$ need not be definable: Take $N$ to be an algebraic subgroup of infinite index in $\mathrm{GL}(V)$ for some finite-dimensional vector space $V$ such that $V$ is $N$-minimal. Let $x \in \mathrm{GL}(V)$ have infinite order modulo $N$, and consider the definable set $S=N \cup\{x\} . N$ has infinite index in the group $G$ generated by $S$, but finite index in any product of a finite number of copies of $S$. Therefore, $G$ cannot be definable. Of course, $N$ is definable and almost contains $S$, and this is, in fact, the general situation: Again by the indecomposability theorem, for any $S$ as in the theorem there is a definable subgroup $G^{\prime}$ of $\langle S\rangle$ such that $S$ is contained in finitely many cosets of $G^{\prime}$ (namely, the stabiliser of a type of maximal rank in a finite power of $S$ of maximal rank).

Thus by Theorems 3 and 4 , an infinitely definable group $G$ of quasi-automorphisms of a $G$-minimal abelian group of unbounded exponent is a linear group.

Note that in Theorem 4, we could not have done with endomorphisms instead of automorphisms: If $R$ is any nilpotent ring of finite rank and $A \leq R^{+}$is $R^{\times}$minimal, then we still cannot define a field.

Corollary 5. In finite Morley rank, a simple group $G$ acting as group of automorphisms on a divisible abelian group $A$ is a subgroup of $G L_{n}(K)$ for some definable algebraically closed field $K$ and some $n<\omega$.

Proof. First, as $A[n]$ is finite for all $n<\omega$, all definable subgroups of $A$ are divisible as well. Let $A_{0} \leq A$ be a minimal $G$-invariant subgroup, so its stabiliser $C$ is a normal subgroup of $G$. If $C=\{1\}$, we are done by Theorem 4, otherwise consider $A_{1}=C_{A}(G) \geq A_{0}$. As $G$ is a group of automorphisms, $C_{G}(A)=\{1\}$ and hence $A_{1}$ is a proper $G$-invariant subgroup of $A$. Let $A_{2} / A_{1}$ be a minimal $G$-invariant subgroup of $A / A_{1}$. Again, either $C_{G}\left(A_{2} / A_{1}\right)=\{1\}$ and we finish by Theorem 4 , or $A_{2} / A_{1}$ is fixed by $G$, that is, $(G-\text { id })^{2}=0$ in End $\left(A_{2}\right)$. But now for any $g, h \in G$,

$$
h g-h-g+1=(h-1)(g-1)=0=(g-1)(h-1)=g h-h-g+1
$$

in End $\left(A_{2}\right)$, hence $h g(a)=g h(a)$ for all $a \in A_{2}$, and $[g, h] \in C_{G}\left(A_{2}\right)$. But this is as a normal subgroup, which cannot be $G$ as $A_{2}>A_{1}$, hence it is trivial. So $G$ is commutative, which is clearly absurd.

However, we cannot prove that $G$ is actually algebraic. The main obstacle is the possible existence of a bad field, i.e., a field $F$ of finite Morley rank with a proper infinite multiplicative subgroup $M$. $M$ will not be an algebraic group, but generates $K$ additively by Zil'ber's indecomposability theorem.

The immediate questions is, of course, what happens in nonzero characteristic? One might probably do something in this case if one assumes the existence 
of a generalised Frobenius automorphism, i.e., an automorphism of $A$ of infinite order, but invariant under $G$. However, the general case does not seem very hopeful.

\section{ACKNOWLEDGMENT}

The first author would like to thank Alistair.

\section{REFERENCES}

[Ch] G. Cherlin, Groups of small Morley rank, Ann. Math. Logic 17 (1979), 1-28.

[C] L. J. Corredor, Gruppen von Endlichem Morley Rang, Ph. D. Thesis, Bonn, 1988.

[Be] C. Berline, Superstable groups, Ann. Pure Appl. Logic 30 (1986), 45-61.

[B] S. Buechler, The geometry of weakly minimal types, J. Symbolic Logic 50 (1985), 10441053.

[H1] E. Hrushovski, Contributions to stable model theory, Ph. D. Thesis, Berkeley, 1986.

[H2] _ Locally modular regular types, Classification Theory, Proceedings, Chicago 1985 (J. T. Baldwin, ed.), Lecture Notes in Math., vol. 1292, Springer-Verlag, Berlin and New York, 1987, pp. 132-164.

[IO] S. V. Ivanov and Y. Ol'shanskiï, Some applications of graded diagrams in combinatorial group theory, London Math. Soc. Lecture Note Ser., vol. 160, Cambridge Univ. Press, Cambridge and New York, 1991, pp. 258-308.

[P1] B. Poizat, Groupes stables, Nur al-Mantiq wal-Ma'rifah, Villeurbanne, 1987.

[P2] _ _ A propos groupes stables, Logic Colloquium '85 (The Paris Logic Group, ed.), North Holland, Amsterdam, 1987, pp. 245-265.

[Re] J. Reineke, Minimale gruppen, Z. Math. Logik Grundl. Math. 21 (1975), 357-359.

Department of Mathematics and Statistics, McGill University, 805 Sherbrooke St. West, Montreal, QC, Canada H3A 2K6

E-mail address: loveys@triples.Math.McGill.CA

Mathematisches Institut, Abteilung für Logik, Albertstrasse 23B, 7800 Freiburg, DEUTSCHLAND 